\title{
Analysis of Carding Actions
}

\author{
Part 2: An Estimation of the Degree of Opening and Productivity \\ By Hiraku Takeda and Hiroyuki Ogawa, Members, TMSJ
}

Faculty of Engineering, Yamagata University, Yonezawa, Yamagata-ken

Based on the Journal of the Textile Machinery Society of Japan, Transactions, Vol.25, No. 6 T97-106(1972)

\begin{abstract}
Attempts have been made to propose a quantitative analysis of the relation between the productivity and the degree of opening, which is closely related to the arrangement of carding points and the operating conditions of a card. The following two assumptions were made to solve the problem easily; (1) a fiber tuft reaching a carding point is simply divided into two smaller tufts so that the proportion in weight of these tufts is equal to the value of the transfer coefficient, and (2) the degree of opening of web can be estimated by the mean value of logarithm of reciprocals of the weight of delivered tufts.

The distribution of delivered tuft weight, the distribution of the number of passings through the carding point and the degree of opening have been derived as the function of the transfer coefficient by these assumptions.

Then, taking account of the experimental results that the productivity is restricted by the fiber load on the cylinder from the standpoint of nep formation, the relation between the degree of opening and the productivity has been discussed.
\end{abstract}

KEY WORDS: CARDING, CARDING ACTION, CARD CLOTHING, CARDS, CARDING EFFICIENCY, PRODUCTION

\section{Introduction}

The tuft-opening on a card is performed by the repeated action of carding points on tufts of fibers. Thus, for better opening to separate a tuft into each fiber it is necessary to increase first the number of actions a tuft receives at carding points before it is transferred to the doffer. On the other hand, this is liable to deteriorate the quality of a web, e. g., fatigue and damage of fibers, nep count, etc.

The deterioration will be remedied without decreasing the opening effect at the cost of productivity, but this is clearly the most unskillful way although the easiest. Consequently, various card design and the arrangement of the working conditions aim at the increase of productivity as much as possible while the contradictory influence due to the number of actions is adjusted.

The quantitative consideration to this problem needs to evaluate the function that the carding point plays in productivity and opening. Martindale ${ }^{[1]}$ first discussed the function of the carding point, defining the fraction of the fibers transferred to the worker or the doffer. Thereafter, many studies on carding has been carried out with this concept. For example, Richards ${ }^{[2]}$ attempted to predict the opening effect by the "delay factor'" Monfort ${ }^{[3]}$ analized the frequency a fiber passes between each carding point. These studies are very suggestive for the problem at present.

The authors' previous paper ${ }^{[5]}$ treated the leveling effect on the roller card. The present paper attempts to clarify the relation between the opening effect and the productivity, by proposing the estimation functicn of the opening effect in term of a delivered tuft weight.

\section{Idealized Opening Action}

A "tuft of fibers" mentioned here is defined as the fiber assembly containing no direct information on the cohesion force among fibers in it. A "fed tuft" is definc $d$ as the fiber assembly that is drawn out from a lap with a taker-in.

Each fed tuft reaching a carding point is divided into two smaller parts and is partly spread on the cylinder surface. The spreading action may be caused by the fact that fibers are partly returned to the cylinder surface again after they have been transferred to the roller. So the fiber-amount returned is strongly affected by the duration in which fikers stay on the carding point.

On a flat card, it is proper to treat the opening action only by the concept of the spreading action, because the 
duration above mentioned continues until fibers on the flats are fully spread on the cylinder surface or removed as flat strips. Contrary to this, in the ordinary operation of the worker or the doffer which runs faster than the flat, the spread fibers are hardly observed due to their short staying time. Really, there was a neglegible amount of them in some experiments. So, in this study it is assumed that the opening action on the roller card is only caused by the dividing action, and that the weight of the tuft divided and transferred to the worker or the doffer is proportional to the transfer coefficient which is independent of an original tuft weight. Considering only one fiber, it may be allowed that this coefficient is equal to the probability that the fiber is transferred to the worker or the doffer.

The theoretical result will be deduced by tracing all the tufts, produced from a fed tuft, until they are transferred to the doffer. The traced tuft, hereafter called the "delivered tuft', means the fiber assembly which were contained in the same fed tuft and passed through the same route.

\section{Distribution of Delivered Tuft Weight}

Fig. 1 illustrates the construction of the roller card, the notations of carding points and transfer coefficients, the taker-in part being omitted. Consider the event that an arbitrarily chosen fiber in a fed tuft passes through any one of the conditioned route, defined by $\left(m_{1}, m_{2}, \ldots, m_{n}, k\right)$ where $m_{1}$ is the number of passings the fiber is transferred to the first worker, $m_{2}$ being that to the second worker, $k$ being that through the carding point $\mathrm{D}$, regardless of the sequence of the transfer.

The probability of this event is easily found as:

$$
\begin{aligned}
& P_{W 1}^{m_{11}}\left(1-P_{W 1}\right) P_{W 2}^{m_{21}}\left(1-P_{W_{2}}\right) \cdots \cdots P_{W n}^{m_{n_{1}}}\left(1-P_{W n}\right) \\
& \cdots \cdots\left(1-P_{D}\right) P_{W 1}^{m_{1 k}}\left(1-P_{W_{1}}\right) P_{W_{2}}^{m_{2 k}}\left(1-P_{W_{2}}\right) \cdots \cdots \\
& P_{W n}^{m_{n k}}\left(1-P_{W n}\right) P_{D} \cdots \cdots \cdots \cdots \cdots(1)
\end{aligned}
$$

were $P_{W i}$ is the transfer coefficient of the $i$ th worker, $P_{D}$ being that of the doffer, The exponent $m_{i j}$ of $P_{W i}$ is the number of passings that the fiber which had passed $(j-1)$ times through the point $\mathrm{D}$, is successively transferred to the $i$ th

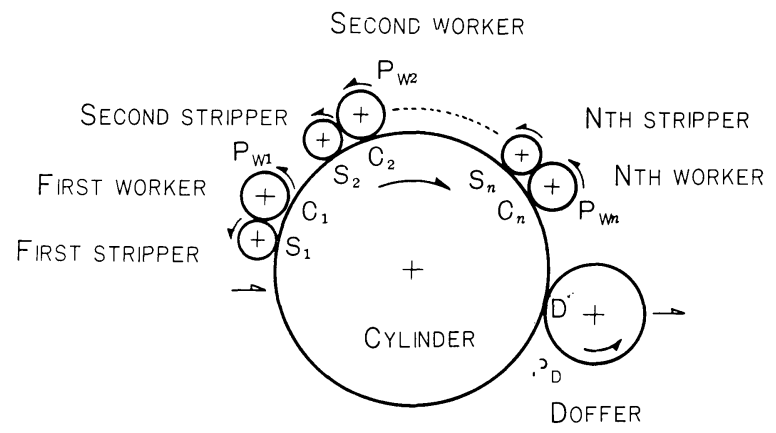

Fig. 1 Schematic diagram of roller card considered worker. Thus the final number of the transfer to the $i$ th worker, $m_{n}$, can be expressed by

$$
m_{i}=\sum_{j=1}^{k} m_{i j} \quad(i=1,2, \cdots \cdots, n) \cdots \cdots \cdots
$$

If the set $\left(m_{1}, m_{2}, \ldots, m_{n}, k\right)$ is given eq. (1) becomes constant, regardless of each $m_{i j}$. Thus eq. (1) is rewritten as

$$
\begin{aligned}
g\left(m_{1},\right. & \left.m_{2}, \cdots \cdots, m_{i}, \cdots \cdots, m_{n}, k\right) \\
& =\frac{P_{D}}{1-P_{D}}\left[\left(1-P_{D}\right) \prod_{i=1}^{n}\left(1-P_{W i}\right)\right]^{k} \prod_{i=1}^{n} P_{W i}^{m i}
\end{aligned}
$$

The probability, such that an arbitrarily chosen fiber in a fed tuft is transferred to the worker or to the doffer by one division, is proportional to the weight of the divided tuft to be transferred. When every tuft weighs equally, the value $\mathrm{g}$ is proportional to the weight of the delivered tuft which has passed through the conditioned route.

"Any one of the conditioned route"' above-mentioned means that the set $\left(m_{1}, m_{2}, \ldots, m_{n}, k\right)$ cannot determine a fixed route. Namely, there are routes as many as the aggregates of possible arrangements of $m_{i j}$ satisfying eq. (2) for all possible $i$. By referring to the form of eq. (1), the arrangements for a fixed $i$ are easily found as $\left(\begin{array}{c}m_{i}+k-1 \\ m_{i}\end{array}\right)$. Since this combination is independent of different $i$, the total number of the conditioned route is obtained as follows:

$$
L\left(m_{1}, m_{2}, \cdots, m_{i}, \cdots, m_{n}, k\right)=\stackrel{n}{I I}\left(\begin{array}{c}
m_{i}+k-1 \\
m_{i}
\end{array}\right)
$$

The product of $L$ and $g$, therefore, gives the probability that a fiber passes through any of the conditioned route:

$$
\operatorname{Pr}\left(m_{1}, m_{2}, \cdots, m_{i}, \cdots, m_{n}, k\right)=L g \cdots \cdots \cdots
$$

In other words, this probability means the weight proportion of a delivered tuft which passed through any of the conditioned route, when every fed tuft weighs equally.

By calculating eq. (4) for all possible routes and classifying their values with regard to the weight which appears in each route, the distribution (on a weight basis) of the delivered tuft weight is obtainable. When each fed-tuft weighs differently, the distribution is obtained by repeating the similar procedure for each fed-tuft weight.

\section{Number of Passings through Carding Point and Transference to Worker}

The number of passings that a fiber passes the carding point, and the frequency that a fiber is transferred to the worker, will be discussed. These are important not only for the analysis, shown in the next chapter but also for an approximate insight into the opening effect.

Consider the probability $f_{W}\left(m_{i}\right)$ with which $m_{i}$ occurred. Since the numbers excluding $m_{i}$ are arbitrary in eq. (4), this 
probability in expressed by:

$$
\begin{aligned}
& f_{W}\left(m_{i}\right)=P_{r}\left(0 \leqq m_{\mathbf{1}}<\infty, 0 \leqq m_{\mathbf{2}}<\infty, \cdots, m_{i}=m_{i},\right. \\
& \left.\cdots, 0 \leqq m_{n}<\infty, 1 \leqq k<\infty\right) \\
& =\sum_{k=1}^{\infty} \sum_{j=1}^{n} \underset{j}{\stackrel{\infty}{N}} \underset{m_{j}=0}{\operatorname{Lg}} \underset{j=1,2, \cdots, i-1, i+1, \cdots, n)}{ } \\
& =\frac{P_{D}}{1-P_{D}} P_{W i}^{m_{i}} \sum_{k=1}^{\infty}\left(\begin{array}{c}
k+m_{i}-1 \\
m_{i}
\end{array}\right) \\
& \left\{\left(1-P_{D}\right)\left(1-P_{W i}\right)\right\} \stackrel{k}{k} I_{j=1}^{n} \sum_{\substack{j \neq i \\
\infty}, j=0}\left(\begin{array}{c}
k+m_{j}-1 \\
m_{j}
\end{array}\right) \\
& \left(1-P_{W j}\right)^{k} P_{w_{j}^{m}}^{m}
\end{aligned}
$$

Simplifying this, (Note 1)

$$
f_{W}\left(m_{i}\right)=\frac{1}{1+N_{W i}}\left(\frac{N_{W i}}{1+N_{W i}}\right)^{m_{i}}
$$

where $N_{W i}$ is the average number of the transference to the $i$ th worker:

$$
N_{W i}=\sum_{m i=0}^{\infty} m_{i} f\left(m_{i}\right)=\frac{P_{W i}}{P_{D}\left(1-P_{W i}\right)}
$$

The average of the total number of the transference on all workers, $N_{W}$ is equal to the sum of eq. (7) for all $i$, and

$$
N_{W}=\sum_{i=1}^{n} N_{W i}=\frac{1}{P_{D}} \sum_{i=1}^{n} \frac{P_{W i}}{\left(1-P_{W i}\right)} \cdots \cdots \cdots \text { (8) }
$$

Now, consider the probability $f_{C}\left(K_{i}\right)$ such that a fiber passes $K_{i}$ times through point $C_{i}$. Since $K_{i}$ is equal to the sum of $k$ and $m_{i}$, and in eq. (4), $1 \leqq k_{i} \leqq K_{i}$ and $m_{i}=K_{i}$ $-k$, the probability is calculated from:

$$
\begin{aligned}
& f_{C}\left(K_{i}\right)=P_{r}\left(0 \leqq m_{1}<\infty, 0 \leqq m_{2}<\infty, \cdots, m_{i}=K_{i}\right. \\
& \left.-k, \cdots, 0 \leqq m_{n}<\infty, 1 \leqq k \leqq K_{i}\right) \\
& =\frac{P_{D}}{1-P_{D}} P_{W^{i} i}^{K^{i}} \sum_{k=1}^{K^{i}}\left(\begin{array}{c}
K_{i}-1 \\
K_{i}-k
\end{array}\right)\left\{\left(1-P_{D}\right)\left(1-P_{W i}\right) /\right. \\
& P_{W i}{ }^{k} I_{j=1}^{n} \quad\left(\begin{array}{c}
k+m_{j}-1 \\
m_{j}
\end{array}\right)\left(1-P_{W j}\right)^{k} P_{w_{j}}^{m j} \cdots
\end{aligned}
$$

This is simplified as follows: (Note 2)

$$
f_{C}\left(K_{i}\right)=\frac{1}{N_{C i}}\left(1-\frac{1}{N_{C i}}\right)^{k_{i}-1}
$$

where $N_{C i}$ is the average number of passings through the point $C_{i}$ :

$$
N_{c i}=\frac{1}{P_{D}\left(1-P_{W i}\right)}
$$

Since the average of the total number of passings through the carding points excepting $D, N_{C}$ is the sum of eq. (11) for all possible $i$,

$$
N_{C}=\sum_{i=1}^{n} N_{C i}=\frac{1}{P_{D}} \sum_{i=1}^{n} \frac{1}{\left(1-P_{W i} i\right)}
$$

In particular, the average number of passings through point $D$ implies $P_{W i}=0$ in eq. (11), so that the total number of passings including point $D$ becomes :

$$
N_{T}=N_{C}+\frac{1}{P_{D}}
$$

Fig. 2 shows the typical curves of eq. (6), the monotonous

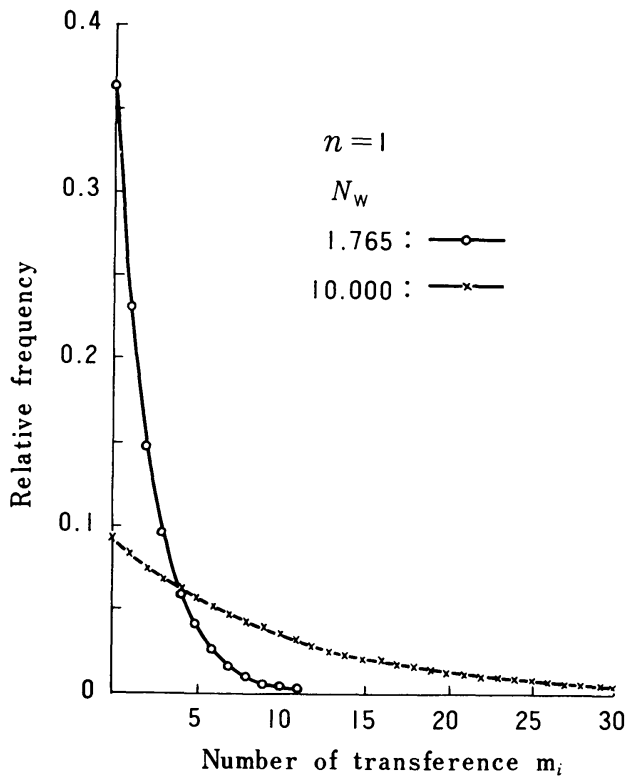

Fig. 2 Distribution of number of transference to $i$ th worker, which corresponds to that on a single worker

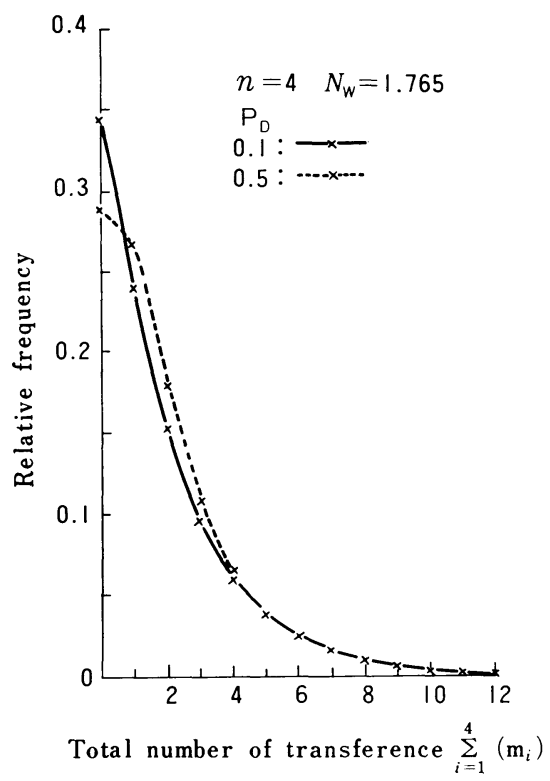

Fig. 3 Distribution of the total number of transference in case of four workes, each of which has the same transfer coefficient

decrease of which suggests the difficulty of achieving a better opening effect with a single worker. In the case of many workers, the distribution of the total number can not be expressed by a simple form. Therefore, the direct numerical calculation of eq. (5) is preferable to draw the distribution curve. 


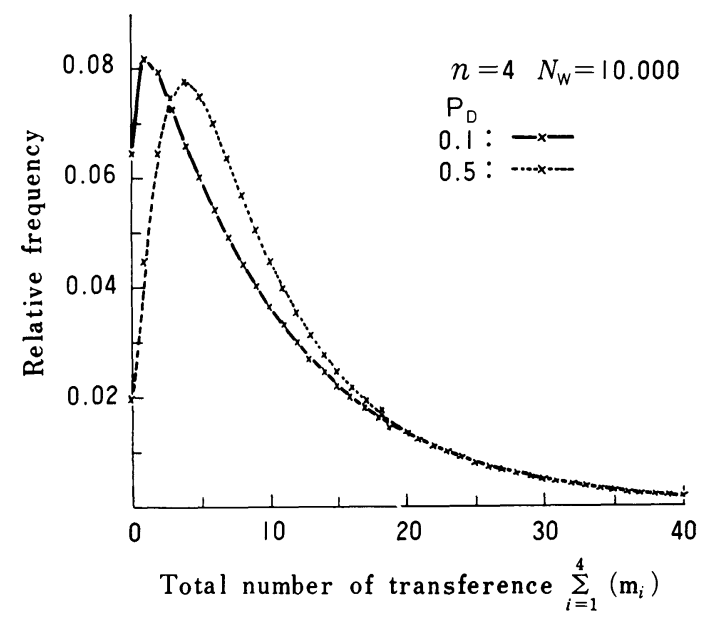

Fig. 4 Distribution of the total number of transference in case of four workrs, each of which has the same transfer coəffficient

Figs. 3 and 4, thus drawn, show the distribution of $\sum m_{i}$ in the case of four workers, each of which has the same transfer coefficient. They show that the number of workers exert marked influence upon the shape of the distribution curve only when $N_{W}$ is large. The peak value, desirable to decrease unopened tufts, appears there.

The variance $V\left(\sum m_{i}\right)$ of $\sum m_{i}$ offers a rough measure of excessive action on a fiber. This is given by: (Note 3 )

$$
V\left(\Sigma m_{i}\right)=N_{W}\left(N_{W}+1\right)-P_{D} \sum_{i, j=1(i \neq j)}^{n} N_{W i} N_{W j}
$$

In the similar way, the variance $V\left(\sum K_{i}\right)$ of $K_{i}$ which is the total number of passings through the carding points excepting point $\mathrm{D}$, is given by:

$$
V\left(\Sigma K_{i}\right)=N_{C}\left(N_{C}+1\right)-P_{D} \sum_{i, j=1 ; i \neq j,}^{n} N_{C i} N_{C j}
$$

The second terms of eqs. (14) and (15) must be zero in a single worker. When the average number of $N_{C}$ or $N_{W}$ remains constant, the variance decreases with the increase of $\boldsymbol{P}_{D}$ or the number of workers and with the decrease of difference among the transfer coefficients of the workers. No difference among the coefficients leads the second term of eq. (14) to:

$$
2 P_{D}\left(\begin{array}{c}
n \\
2
\end{array}\right) \frac{N_{W^{2}}}{n^{2}}=\frac{n-1}{n} P_{D} N_{W}^{2}
$$

In case of more than four workers, this value changes little, and also the shape of the distribution curve of $\sum m_{i}$ will be so. This may be the reason for using usually three or four workers.

\section{Estimation of Opening Effect}

The estimation function of the opening effect in term of an delivered tuft weight is proposed here. In spite of the importance of this subject for full understanding of carding, such an attempt has not yet been done. It would be natural to consider that the opening effect by one division is kept constant irrespective of the original tuft weight, and that the existance of a large delivered tuft in the web, even if rarely contained, brings remarkable deterioration to the opening effect. The following is based on this acceptable consideration.

\subsection{Degree of Opening}

If the transfer coefficient is $(1 / 2)$, the original tuflt is divided into equal small parts weighing proportional to $(1 / 2)^{n}$ by $n$ division, and the opening effect can be estimated by $n$, provided that a unit value is assigned to the effect by one division. In addition the farther the transfer coefficient deviates from $1 / 2$, the more irregular the weight of divided tufts, resulting in the low opening effect. Thus the following estimation function may be presented as one of the useful measure of the opening effect:

$$
S=E\left[\log _{2}(1 / g(]\right.
$$

where $E$ is the operator to evaluate the average basad on eq. (14), $S$ being hereafter called the degree of opening.

By applying eqs. (3) and (4) to eq. (16), the following is obtainable:

$$
S=\frac{1}{P_{D}} \varepsilon_{D}+\sum_{i=1}^{n} \frac{1}{P_{D}\left(1-P_{W i}\right)} \varepsilon_{W i} \cdots \cdots
$$

where $\varepsilon_{D}$ and $\varepsilon_{W i}$ are given respectively by:

$$
\begin{aligned}
& \varepsilon_{D}=P_{D} \log _{2} \frac{1}{P_{D}}+\left(1-P_{D}\right) \log _{2} \frac{1}{1-P_{D}} \\
& \varepsilon_{W i}=P_{W i} \log _{2} \frac{1}{P_{W i}}+\left(1-P_{W i}\right) \log _{2} \frac{1}{1-P_{W i}}
\end{aligned}
$$

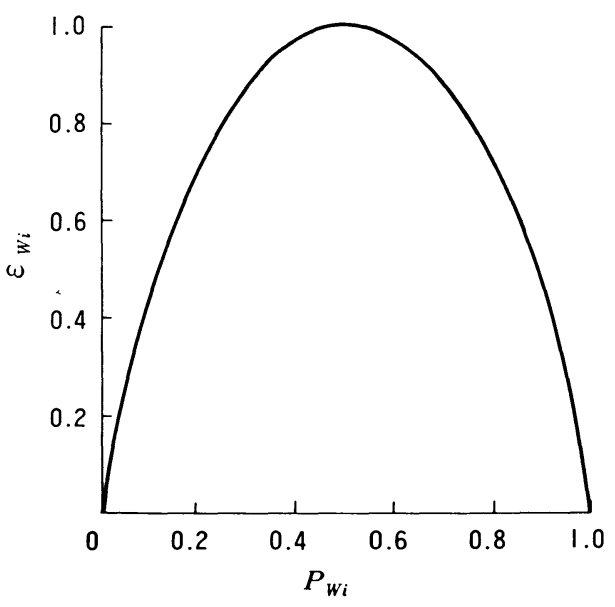

Fig. 5 Relation between transfer coefficient and degree of opening caused by one division

Journal of The Textile Machinery Society of Japan 


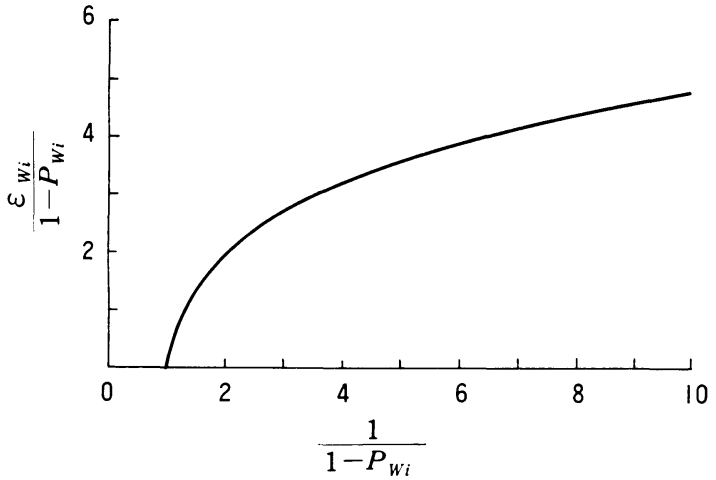

Fig. 6 Degree of opening caused by one worker

As shown in eq. (11), the coefficient of $\varepsilon_{D}$ is the average of passings the tuft passed through point $D$, the coefficient of $\varepsilon_{W i}$ being that of point $C_{i}$, respectively. So, $\varepsilon_{D}$ gives the degree of opening due to one division at point $D, \varepsilon_{W i}$ giving that at point $C_{i}$ as shown in Fig. 5. These values decrease symmetrically as the transfer coefficient deviates from $1 / 2$.

Fig. 6 shows the relation between $\varepsilon_{W i} /\left(1-\boldsymbol{P}_{W i}\right)$, expressing the increment of $S$ at point $C_{i}$, and $1 /\left(1-P_{W i}\right)$ which is proportional to the average number of division. $\varepsilon_{W i}$ decreases when $P_{W i}$ exceeds $1 / 2$, but the increment of $S$ increases consistently due to the rapid increase of the average number of division. This results in the fact that, under a fixed number of passage no difference of the transfer coefficients among the workers makes the degree of opening maximum as predicted in the previous Chapter.

In Fig. $7 \boldsymbol{P}_{D}$ is plotted against $\boldsymbol{P}_{W i}$ for various $\mathrm{S}$ : the dotted line shows the case of a single worker, the full line

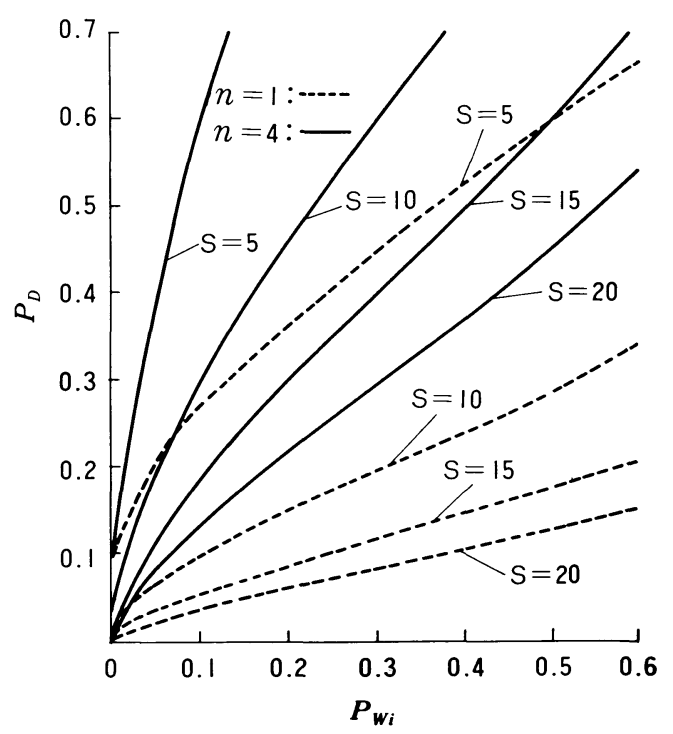

Fig. 7 Relation between $P_{D}$ and $P_{W i}$ for various degree of opening the case of four workers, each of which has the equal transfer coefficient. This relationship is useful to find the way of deciding the proper transfer coefficient under a given $\mathbf{S}$.

\subsection{Moment of Delivered Tuft Weight}

The delivered tuft weight itself will be studied here, instead of the number of passings, for dealing with the degree of opening. Namely, is considered the higher order moment of the delivered tuft weight, which is used to estimate the degree of unopening. The $\mu$ th order moment about the origin, $M(\mu)$ is expressed by: (Note 5)

$$
\begin{aligned}
M(\lambda) & =\sum_{k=1}^{\infty} \sum_{m i=0}^{\infty} L g^{\prime \prime} \\
& P_{D}^{\mu} \prod_{i=1}^{n}\left(1-P_{W i}\right)^{\mu} \\
= & \left.I_{i=1}^{n}\left(1-P_{w^{\mu} i}^{\mu}\right)-\left\{1-P_{D}\right) \prod_{i=1}^{n}\left(1-P_{W i}\right)\right\}^{\mu}
\end{aligned}
$$

where $M(2)$ shows the average weight on a weight basis.

On account of the defficiency of experimental work at present, it is difficult to say which gives more accurate measure, $S$ or $M(\mu)$. However, it is sure that the value $S$ gives the intuitive perspective and is convenient to use for the complicated process such as the series of card operation, because it has the close connection to the average number of passings and shows the additive rule as to the effect of each carding point.

\section{Relation between Degree of Opening and Producti- vity}

The degree of opening is unlimitedly improved by increasing the total number of passings through the carding point. As clarified in Chapter 4 , the easist method to increase this degree is to reduce $P_{D}$. But it is wall known that this way is undesirable for both the productivity and other web qualities except the degree of opening.

The author ${ }^{[4]}$ showed experimentally that the fiber length decrease due to its breakage was strongly affected by the number of passings on the roller card where fibers were repeatedly carded under fixed operating conditions. This result should be regarded as the manifestation of the harmful influence of the number of passings when the fiber load per unit area of the cylinder surface is kept constant.

At a constant rate of production, the average number of passings through the $i$ th carding point, $N_{C i}$, is proportional to the fiber weight to be transported there in unit time. The fiber load at the $i$ th carding point is therefore equal to the ratio of $N_{C i}$ to the surface speed of the cylinder. If there is an allowable fibs load to be decided from the amount of the harmful influence, then the production rate must be adjusted in accordance with the change in $N_{C i}$. Namely, 


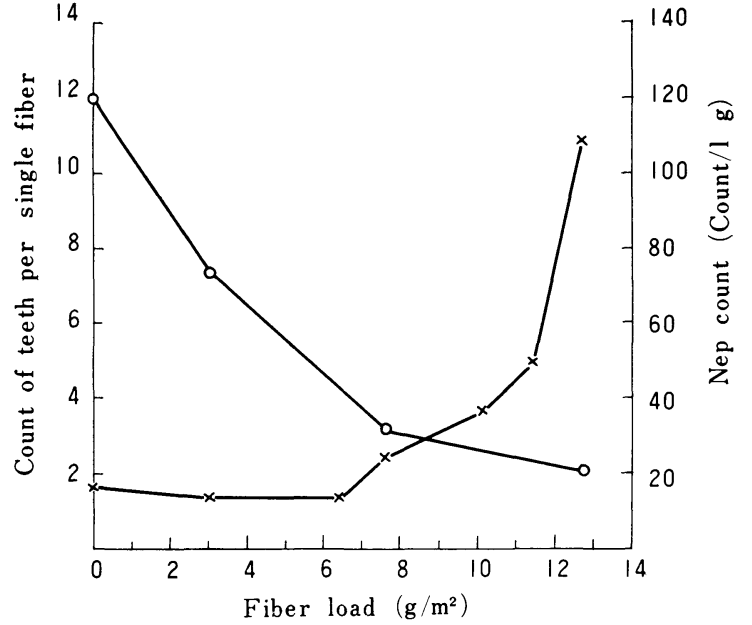

Fig. 8 Nep count $(-\times-)$, and average teeth count $(-\mathrm{O}-)$ plotted against fiber load on cylinder surface (Gauge between cylinder and worker is $10 / 1,000 \mathrm{in}$.)

not only the total mnumber of $N_{W i}$ but also $N_{C i}$ are considerably important.

To clarify the harmful influence of the fiber load, the nep count in $1 \mathrm{~g}$ of fibers, crimped 2 den. viscose staples with $57 \mathrm{~mm}$ length, was examined. Weighted fibers were fed to the card consisting of a cylinder, worker and stripper, (without doffer). After the cylinder had been rotated exactly by two hundred revolutions, neps were counted.

Mark $\times$ in Fig. 8 shows the nep count plotted against the fiber load on the cylinder. The count at zero load shows the initial count contained in the sample fibers carded beforehand. The figure clarifies that the fiber load has no effect on the nep formation up to about $6 \mathrm{~g} / \mathrm{m}^{2}$ but that it

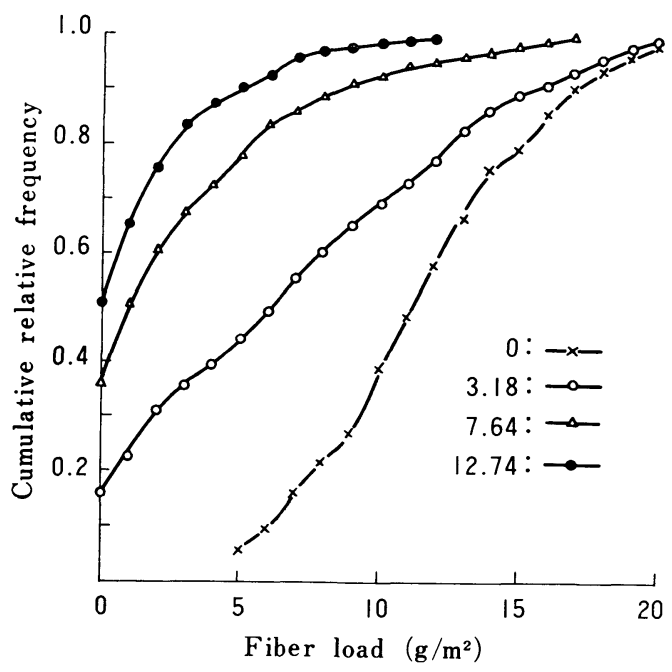

Fig. 9 Cumulative relative frequency of teeth count exerts much influence on the nep formation beyond this boundary.

Mark $\bigcirc$ in Fig. 8 shows the average count of the card wire teeth which hold a single fiber.

To facilitate the observation, several tens of fibers dyed with fluorescent dyestuff were added to the sample fibers. The tracer fibers on the cylinder, stopped after two hundred revolutions, were observed obliquely, and the number of teeth hindering the observation of the noted fiber was counted. Such a count would not precisely agree with the real count of teeth holding a fiber, but undoubtedly it is a good approximate measure of that count.

The cummulative relative frequency of the teeth count is shown in Fig. 9, which shows the high dependence of nep fromation upon the amount of fibers free or nearly free from card wire teeth. This experiment seems to support that there is an allowable fiber load clearly define from the point of the harmful influence of carding.

Fig. 10 shows the relationship between $S$ and $N_{C i}$, Figs. 11 and 12 being the second and the fourth order moment, respectively, in which $n$ is the number of workers and each mark $\times$ shows the value corresponding to $P_{W i}=0$ for all $i$. As shown in these figures, the larger $P_{D}$, the worse the opening. But this tendency is overcome by the proper increase of the number of workers. The increase of workers generally reduces $N_{C i}$ required to perfrom a given degree of opening; this is very important for the productivity.

The usefulness of the results obtained so far may be verified by the next example. Consider the cases of $P_{D}=0.1$ and $P_{D}=0.5$, at $n=4$, and let the required $S$ be 15 . Then

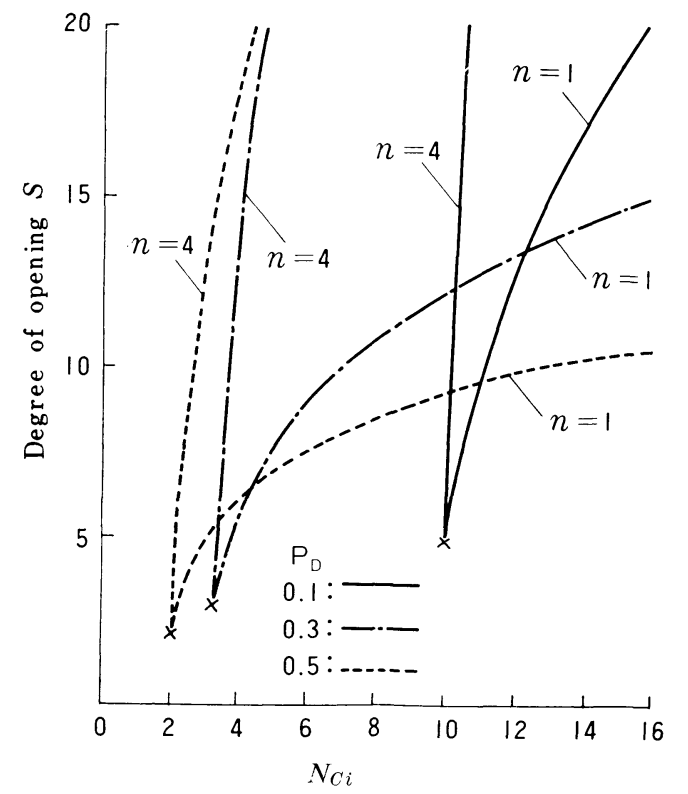

Fig. 10 Relation between degree of opening $S$ and number of passage through $i$ th carding point, $N_{C i}$ 


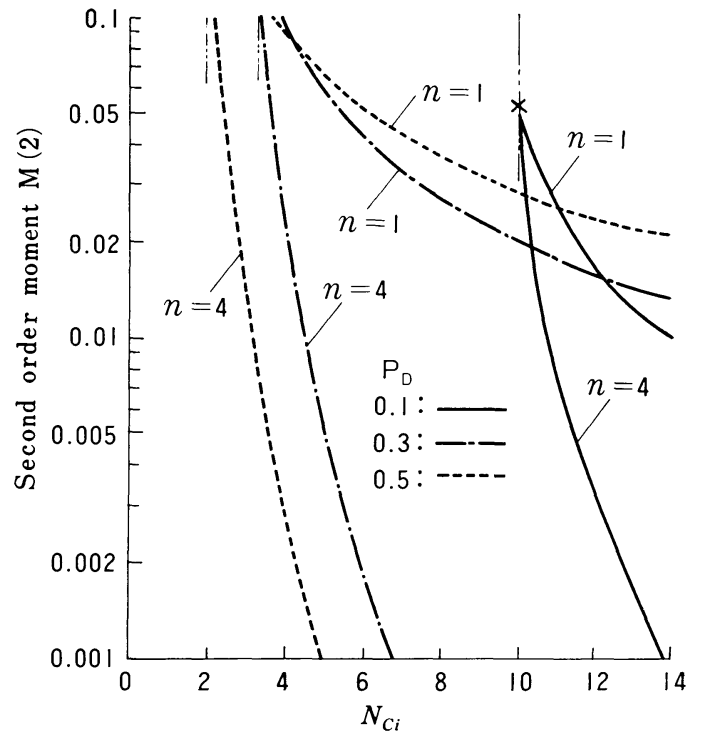

Fig. 11 Relation between second order moment $\mathrm{M}(2)$ and number of passage, $N_{C i}$, through $i$ th carding point

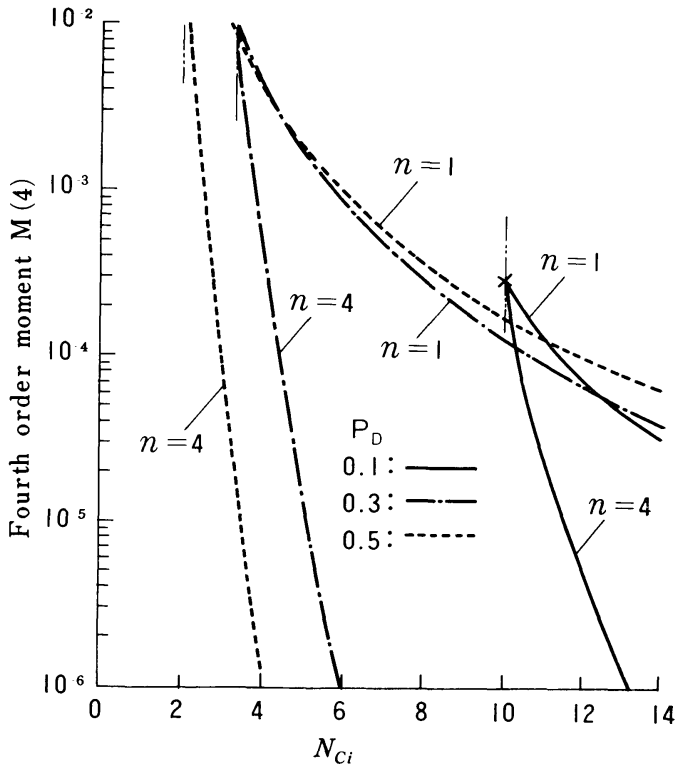

Fig. 12 Relation between fourth order moment M(4) and number of passage, $N_{C i}$, through $i$ th carding point

Fig. 10 shows that the former case requires $N_{C i}$ nearly three times larger than the latter case. In other words, if the fiber load can be allowed up to the value of the forme case, then the productivity of the latter case can be improve 3 times without trouble. Moreover it is of intersest that the total average $N_{T}$ of the latter case is smaller than that of the case $P_{D}=0.1$ in a single worker, in spite of the larger number of carding points.

\section{Literature Cited}

[1] J. G. Martindale; J. Text. Inst., 36, T213 (1945)

[2] P. T. D. Richards; J. Text. Inst., 50, P182 (1959)

[3] F. Monfort; J. Text. Inst., 53, T379 (1962)

[4] H. Takeda; J. Text. Mach. Soc. Japan, English ed., 15, 214 (1969)

[5] H. Takeda, H. Ogawa; J. Text. Mach. Soc. Japan, 23, T151 (1970)

\section{Note}

(Note 1): The following identity is of considerable importance to work out the mathematical problem of this study:

$$
\sum_{i=0}^{\infty}\left(\begin{array}{c}
n+i \\
i
\end{array}\right) x^{i}=(1-x)-(n+1) \ldots \ldots \ldots \ldots(\mathrm{i})
$$

By using this, eq. (5) can be simplyfied as:

$$
\begin{aligned}
& f_{W}\left(m_{i}\right)=\frac{P_{D}}{1-P_{D}} P_{W i}^{m_{i}} \sum_{k=1}^{\infty}\left(\begin{array}{c}
k+m_{i}-1 \\
m_{i}
\end{array}\right) . \\
& \left\{\left(1-P_{D}\right)\left(1-P_{W i}\right)\right\}
\end{aligned}
$$

Note that $\left(\begin{array}{c}k+m_{i}-1 \\ m_{i}\end{array}\right)=\left(\begin{array}{c}k+m_{i}-1 \\ k-1\end{array}\right)$ and apply again eq.

(i) to eq. (ii). Then the following is obtainable:

$$
\begin{aligned}
& f_{W}\left(m_{i}\right)=\frac{P_{D}\left(1-P_{W i}\right)}{P_{D}\left(1-P_{W i}\right)+P_{W i}} . \\
& \left\{\begin{array}{c}
P_{W i} \\
P_{D}\left(1-P_{W i}\right)+P_{W i}
\end{array}\right\}^{m_{i} i}
\end{aligned}
$$

From this the average of $m_{i}$ is easily found as given in eq. (7). Thus the substitution of eq. (7) into eq. (iii) gives eq. (6).

(Note 2): By using eq. (i), eq. (9) is simplified as:

$$
\begin{aligned}
& f_{C}\left(K_{i}\right)=\frac{P_{D}}{1-P_{D}} \cdot P_{W_{i}}^{K_{i}} \sum_{k=1}^{K^{i}}\left(\begin{array}{l}
K_{i}-1 \\
K_{i}-k
\end{array}\right) \cdot \\
& \left\{\left(1-P_{D}\right)\left(1-P_{W_{i}}\right)\left(1-P_{W_{i}}\right) / P_{W_{i}}\right\} k \ldots \text { (iv) }
\end{aligned}
$$

Note that $\left(\begin{array}{c}K_{i}-1 \\ K_{i}-k\end{array}\right)=\left(\begin{array}{c}K_{i}-1 \\ k\end{array}\right)$ and apply again eq. (i) to eq. (iv). Then the following is obtainable:

$$
\begin{aligned}
& f_{C}\left(K_{i}\right)=P_{D}\left(1-P_{W_{i}}\right)\left\{P_{W_{i}}+\left(1-P_{D}\right) \cdot\right.
\end{aligned}
$$

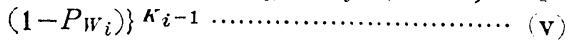

With such a calculation as in (Note 1), eq. (10) is derived from eq. (v).

(Note 3): The definition of the variance gives the following expression:

$$
\begin{aligned}
& V\left(\sum m_{i}\right)=E\left[\left(\Sigma m_{i}\right)^{2}\right]-N_{W}^{2} \\
& =\sum_{i=1}^{n} E\left[m_{i}^{2}\right]+\sum_{i, j=1}^{n} E\left[m_{i} m_{j}\right]-N_{W}^{2} \cdots \cdots(\mathrm{vi})
\end{aligned}
$$

Since the following is easily understood, the first term $o$ : eq. (vi) is :

$$
\begin{aligned}
& E\left[m_{i}^{2}\right]=\sum_{m i=0}^{\infty} m_{i}^{2} f_{W}\left(m_{i}\right)=2 N_{\boldsymbol{W}^{\prime}}{ }_{i}+N_{W_{i}} \\
& \sum_{i=1}^{n} E\left[m_{i}^{2}\right]=\sum_{i=1}^{n}\left(2 N_{W^{2} i}^{2}+N_{W_{i}}\right) \cdots \cdots \cdots \text { (vii) }
\end{aligned}
$$


The s cond term is obtained by calculating the following

$$
\begin{aligned}
& E\left[m_{i} m_{j}\right]=\frac{P_{D}}{1-P_{D}} \sum_{k=1}^{\infty}\left\{\left(1-P_{D}\right)\left(1-P_{W_{i}}\right) \cdot\right. \\
& \left.\left(1-P_{W j}\right)\right\}^{k} \sum_{m_{i}=0}^{\infty} m_{i}\left(\begin{array}{c}
k+m_{i}-1 \\
m_{i}
\end{array}\right) P_{W_{i}}^{m_{i}} . \\
& \sum_{m_{j}=0}^{\infty} m_{j}\left(\begin{array}{c}
k+m_{j}-1 \\
m_{j}
\end{array}\right) P_{m_{j}}^{w_{j}}
\end{aligned}
$$

Thə differentiation of eq. (i) gives

$$
\sum_{i=0}^{\infty} i\left(\begin{array}{c}
n+i \\
i
\end{array}\right) x^{i}=x(n+1)(1-x)-(n+2)
$$

So, eq. (viii) becomes

$$
E\left[m_{i} m_{j}\right]=\left(2-P_{D}\right) N_{W i} N_{W j}
$$

From these results eq. (14) is obtainable.

(No.e 4): Substituting eq. (3) into eq. (16),

$$
\begin{aligned}
& S=E\left[\log _{2} \frac{1-P_{D}}{P_{D}}+k \log _{2} \frac{1}{1-P_{D}}\right. \\
& \left.+k \sum_{i=1}^{n} \log _{2} \frac{1}{1-P_{W i}}+\sum_{i=1}^{n} m_{i} \log _{2} \frac{1}{P_{W i}}\right] \\
& =\log _{2} \frac{1-P_{D}}{P_{D}}+\log _{1-P_{D}} \frac{1}{1-k]} \\
& +\sum_{i=1}^{n} \log _{2} \frac{1}{1-P_{W i}} E[k]+\sum_{i=1}^{n} \log _{2} \frac{1}{1-P_{W i}} E[k] \\
& +\sum_{i=1}^{n} \log _{2} \frac{1}{P_{W i}} E\left[m_{i}\right] \cdots \cdots \cdots \ldots \ldots \ldots \ldots \ldots(\mathbf{x})
\end{aligned}
$$

Since the averages of $k$ and $m_{i}$ are $1 / \boldsymbol{P}_{D}$ and $\boldsymbol{P}_{W i} /\left(1-\boldsymbol{P}_{W i}\right)$ $\boldsymbol{P}_{D}$, respectively, eq. (17) is obtainable by substituting them into each term of eq. (x).

(Note 5): By substituting eqs. (3) and (4) into eq. (19), the following is obtainable:

$$
\begin{aligned}
& M(\mu)=\left(\frac{P_{D}}{1-P_{D}}\right)^{\mu} \sum_{k=1}^{\infty}\left(1-P_{D}\right)^{n k} . \\
& \prod_{i=1}^{n} \sum_{m i=0}^{\infty}\left(\begin{array}{c}
k+m_{i}-1 \\
m_{i}
\end{array}\right)\left(1-P_{\boldsymbol{H}_{i}}\right)^{i k} P_{W_{i}}^{m_{i}} \quad \cdots \quad(\mathbf{x i})
\end{aligned}
$$

Applying the identity obtained by replacing $x$ of eq. (i) with $x^{\mu}$, eq. (xi) becomes the following geometric series:

$$
\begin{aligned}
& M(\mu)=\left(\frac{P_{D}}{1-P_{D}}\right)^{\mu} \sum_{k=1}^{j}\left(1-P_{D}\right)^{k k} . \\
& \prod_{i=1}^{n}\left(1-P_{W i}\right)^{\mu k}\left(1-P_{W_{i}}^{\mu}\right)-k \ldots \ldots \ldots . .
\end{aligned}
$$

From this, eq. (19) is obtainable easily. 